\title{
Effects of Unstable Footwear on Stance Pattern
}

\author{
M. A. Choukou, S. Ghouli, F. C. Boyer, R. Taiar \\ Laboratoire de Biomécanique, GRESPI, Université de Reims-Champagne Ardenne, Reims, France \\ Email: choukouamine@gmail.com
}

Received January 2014

\begin{abstract}
Inadequate safety shoe wearing is thought to be responsible of many musculoskeletal disorders. A new concept of unstable footwear is proposed to reduce mechanical stresses during work. Major expectations argue for a better ankle dynamic behavior leading to an increased muscular activity. The aim of this study was to evaluate the variation of stance characteristics while walking on level ground as a function of the models of safety shoes. Twenty healthy males (aged $23.6 \pm 7$ ) were asked to make six gait cycles on a walking track while barefoot, shod with low cost safety footwear, high cost one, and equipped by Masaï Barefoot Technology shoes (the most unstable footwear). Stance phases (heel strike, flat foot and toe-off) have been normalized as percentages of the maximum value of ground reaction force recorded during each step. The highest significant percentages have been observed in Masaï Barefoot Technology shoes condition and only during heel strike (8\% to $10.1 \%$ higher) and toe-off (10\% to $11.3 \%$ higher). In contrast, the temporal durations of the three stance phases were not significantly different between the four conditions. The convexity of safety shoe induces less stability increasing the adaptation of the muscle activity of the wearer at heel-strike and toe-off in order to regain stable gait pattern.
\end{abstract}

\section{Keywords}

Gait, Barefoot, Convex Shoes, Pain

\section{Introduction}

The concepts, methods as well as the analytic techniques that are used to characterize the human mechanics represent major economic challenges. Their development has to be described in a vision of interaction between physical sciences (metrology, systems mechanics and electronics), information technologies and life sciences (materials, tissues, organs and parts) and represent the interface between biomechanics and preventive medicine. Based on the optimization of new materials, the study consists of validating prototypes destined to improve ergonomics at work and to optimize the comfort of the walking users. Walking is one of the most important functions for everyday life as well as specific displacements in some industrial workstations. It is generally the most elementary form of moving. No other movement is performed as automatically as walking. Indeed, it is a complex interaction of selectively controlled muscle activity and joint movements as well as positional perception which allow a person to move at a certain speed in a chosen direction [1]. However, walking could be altered as 
a function of specific shoeing such as safety footwear which is mandatory used in handling workstation. In particular, foot protection is one of the multiple safety procedures which have to be respected by handling professionals. Undoubtedly, safety shoes do protect the feet from mechanical (heavy object falling), thermic, chemical and electrical risks in conformity with ISO 20345 European Norms [2]. However, safety footwear discomfort and fatigue-related effects have been reported by long-time workers [3] suspecting direct relation between shoeing conditions, pain, fatigue and musculoskeletal troubles. Some companies promise that using unstable shoes can alter gait pattern and reduce the harmful effects of stable professional postures or anti-ergonomic locomotion. Evidences of unstable shoes mechanical benefits while walking are questionable since walking is an automatic pattern of serial movements as previously mentioned. The main argue for these expectations is that the unstable-shoes wearer automatically tends to regain a biomechanically stable pattern while standing [4] or walking [5] [6] [7], which force him to increase muscular activity of the lower limb.

The purpose of this paper is to study the effects of wearing unstable shoes on variability of the characteristics of the three stance phases during walking; namely the heel strike, flat foot and toe-off. Without pretending relationship between shoeing conditions and mid- or long-term musculoskeletal troubles in workers, this experiment is aiming to determine whether unstable shoes have specific advantages compared to standard safety footwear.

\section{Methods}

\subsection{Participants and Procedures}

20 healthy males (Table 1) were asked to make six walking cycles (=12 steps) on a walking track (Figure 1) in laboratory conditions. All testing occurred in the morning in order to avoid fatigue effects on walking pattern. None of participants was a handling professional or had lower limb abnormalities according to the medical diagnosis. All participants signed a written consent before taking part to the study. The latter has been conducted in conformity with ethical standards.

The first 30 minutes of the experiment, which lasted approximately one hour of time per person, was dedicated to a familiarization period. Participants had to walk over the walking track according to four conditions and at a preferred frequency until self-reported strange sensations disappeared. Then, three repetitions of each of the following shoeing conditions were recorder (Figure 1).

Table 1. Description of the sample.

\begin{tabular}{cccccc}
\hline \multirow{2}{*}{ Participants } & \multicolumn{4}{c}{ Anthropometric characteristics } \\
\cline { 2 - 5 } & Age & Height & Weight & BMI & Shoe size \\
\hline 20 & $23.6 \pm 7$ & $180 \pm 5.5$ & $78.5 \pm 8.4$ & $42 / 43$ & $24.19 \pm 2$ \\
\hline
\end{tabular}

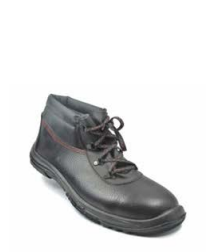

Lambda

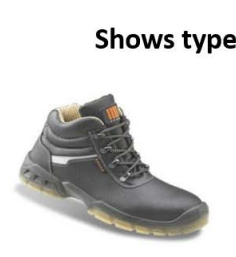

Oregon

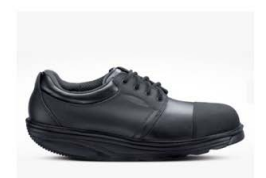

MBT

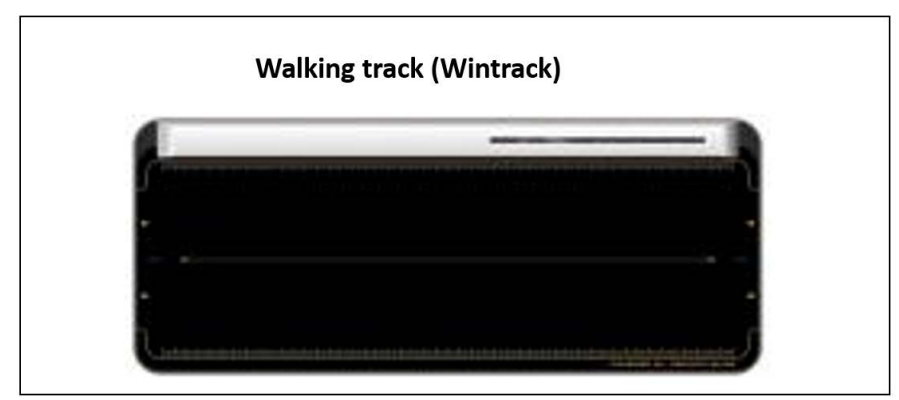

Figure 1. Shoe types and walking track. 
- "Barefoot"

- Shod with a standard and low cost safety shoes ("Lambda")

- Shod with a standard and high cost safety shoes (“Oregon”)

- Shod with a Masaï Barefoot Technology shoes ("MBT")

The main outcome measurements were the amount of ground reaction force (GRF) exerted during the heel strike, flat foot and toe-off phases of each step. The pic GRF of each phase has been further normalized as a percentage (\%) of the pic obtained for the entire step. These stance characteristics have been averaged for all steps of a same trial and for both right and left legs. The corresponding temporal durations have also been took into account as percentage with the same considerations.

\subsection{Measurement Device}

In this study, walking has been evaluated using a walking track, a "Win-Track" (Medicapteurs Technology, Balma, France) platform (length $\times$ width $\times$ height dimensions, $1610 \mathrm{~mm} \times 652 \mathrm{~mm} \times 30 \mathrm{~mm}$; thickness, $9 \mathrm{~mm}$; number of sensors: 12 288, sensor dimension, $7.8 \times 7.8 \mathrm{~mm}^{2}$; acquisition frequency $200 \mathrm{~Hz}$ ) which is a foot pressure sensitive walkway dedicated to static, postural, and gait analysis was used. Walking cycle are deduced from the two steps recorded when the participant walk over the track. A prestructured test protocol was followed to ensure feasibility, accuracy and repeatability of the measurements. Furthermore, the device has been previously validated by Ramachandra et al. (2012) [8] and its use is one of the gait analysis protocols developed in our laboratory of biomechanics [9].

\subsection{Statistical Analyses}

The study design adopted was a single group repeated measures design. All statistical analyses were performed using STATISTICA, version 7 (Statsoft, Tulsa, OK). As data normality was checked, a repeated-measures analysis of variance with Fisher's post-hoc test was performed to establish if there were significant differences between the characteristics of stance phases in function of the four shoeing conditions. The $\alpha$ level was set at 0.05 .

\section{Results}

The results are shown in Figure 2. The main results show that temporal parameters of the stance phases did not change between the three different shoeing conditions. However, the percentages of ground reaction force changed between the four conditions ( $p<0.05$ ). The force of heel strike while equipped by a MBT shoes is 8 to $10.1 \%$ higher than all the conditions. That was also the case for the toe-off values which were 10 to $11.3 \%$ higher for MBT conditions as shown in Figure 2. The results showed the main role of unstable (convex) shoes in increasing the muscle activity of the worker compared to standard safety footwear. Besides, high cost and low cost safety shoes did not show any significant effects on stance parameters. So, it is clear that the safety shoes price cannot insure the better body balance during work.

\section{Discussion and Conclusion}

It is well known that most of handling workers have problems relating to their feet, legs and low back. These problems are highest with workers who are standing many hours per working day. A correlation exists between these complaints and general worker fatigue. Absenteeism and decreased productivity have a negative impact on labour costs and results. Worker injuries could result in (long term) absenteeism, decreased productivity or even liability claims. These factors have a negative impact on costs and yield. The aim of the current study was to determine whether wearing highly unstable safety shoes modify the walking pattern by increasing muscular activity as advocated by the manufacturer. The obtained results showed that when equipped with convex shoes, the feet and therefore also the body move to keep the balance necessary to maintain upright position by increasing the adaptation of the muscle activity. To reiterate, the major outcome of the experiment was that force exerted on the ground during the heel strike is higher while wearing Masaï Barefoot Technology shoes which were the most unstable footwear. This seems to have a blocking effect which results in higher force exerted at the end of the stance while the foot switches from heel strike to toe-off. Surprisingly, the forces observed during flat foot phase remained unchanged and the duration of flat foot did not vary according to this "artificial" swing as one could speculate. These findings are in accordance with the literature [4]-[7] showing the benefits of unstable 


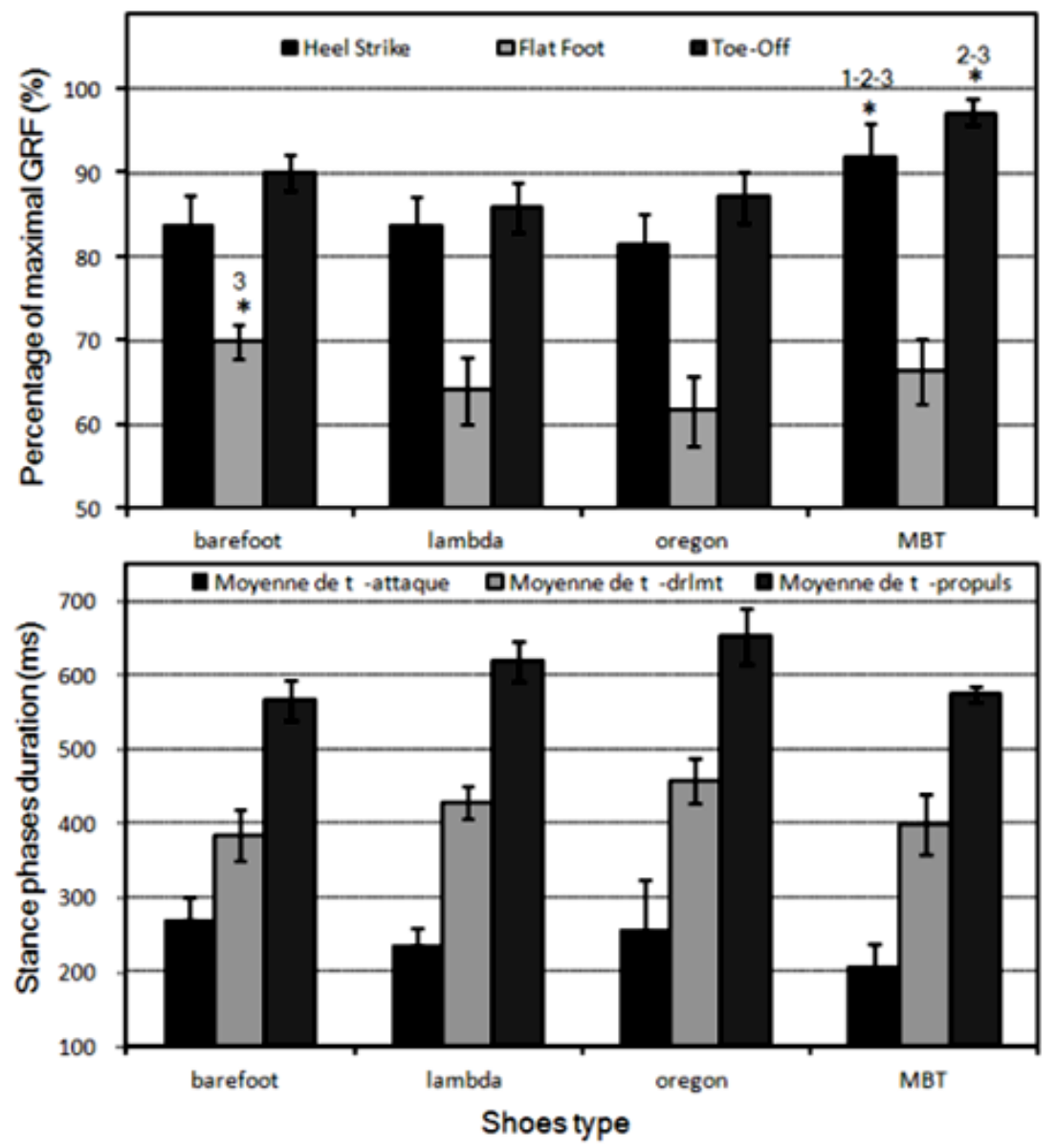

Figure 2. Temporal and force characteristics of the stance phase according to shoeing conditions. $(*)$ refers to significant differences between one or more conditions and the associated number refers to the conditions in left to right order (1 for barefoot and 4 for MBT).

safety shoes on muscle activities of workers. The unexpected result was that high-cost safety shoes do not offer any ergonomic benefits except self-reported comfort sensation compared to low-cost ones. That being said, it is legitimate to question about the possibility of inducing alteration of stance phases as observed in Masaï Barefoot Technology users, by giving instructions for the worker regarding his locomotion behavior (forcing his own heel strike and toe-off reaction forces). The perspective of this research is also to study how safety footwear could help to neutralize machine vibrations in specific workstations.

\section{References}

[1] Goetz-Neumann, K. (2003) Gehen Verstehen. Thieme Verlag.

[2] INRS (2007) Les articles chaussants de protection: Choix et utilisation. Préconisations.

[3] King, P.M. (2002) A Comparison of the Effects of Floor Mats and Shoe In-Soles on Standing Fatigue. Applied Ergonomics, 33 (5), 477-484. http://dx.doi.org/10.1016/S0003-6870(02)00027-3

[4] Landry, S., Nigg, B. and Tecante, K. (2010) Standing in an Unstable Shoe Increases Postural Sway and Muscle Activity of Smaller Extrinsic foot Muscles. Gait \& Posture, 32, 215-219. http://dx.doi.org/10.1016/j.gaitpost.2010.04.018

[5] Price, C., Smith, L., Graham-Smith, P. and Jones, R. (2012) Single-Leg Balance in "Instability” Footwear. 3rd Congress of the International Foot and Ankle Biomechanics Community. Journal of Foot and Ankle Research, 5, 10. http://dx.doi.org/10.1186/1757-1146-5-S1-P10

[6] Nigg, B., Federolf, P., Tscharmer, V. and Nigg, S. (2012) Unstable Shoes: Functional Concept and Scientific Evidence. Footwear Science, 4, 72-73. http://dx.doi.org/10.1080/19424280.2011.653993 
[7] Taniguchi, M., Tateuchi, H., Takeoka, T. and Ichihashi, N. (2012) Kinematic and Kinetic Characteristic of MBT Foot Wear. Gait \& Posture, 35, 567-572. http://dx.doi.org/10.1016/j.gaitpost.2011.11.025

[8] Ramachandra, P., Maiya, A.G. and Kumar, P. (2012) Test-Retest Reliability of the Win-Track Platform in Analyzing the Gait Parameters and Plantar Pressures during Barefoot Walking in Healthy Adults. Foot \& Ankle Specialists, 5, 306-312. http://dx.doi.org/10.1177/1938640012457680

[9] Choukou, M.-A. and Hijazi, S. (2013) Effectiveness of Ankle Taping on Ankle Joint Kinematics during Walking on Level Ground. Foot \& Ankle Specialist, 6, 352-355. 